\title{
Research of Tile Type Transceiver Module Integrating with Two-Dimensional Sum Difference Network
}

\author{
Taifu Zhou, Jian Zhang \\ Southwest China Institute of Electronic Technology, Chengdu, China \\ Email: taifuzhou@163.com
}

How to cite this paper: Zhou, T.F. and Zhang, J. (2021) Research of Tile Type Transceiver Module Integrating with TwoDimensional Sum Difference Network. Journal of Computer and Communications, 9, 116-124.

https://doi.org/10.4236/jcc.2021.912009

Received: September 10, 2021 Accepted: December 28, 2021 Published: December 31, 2021

\begin{abstract}
Transceiver module and two-dimensional sum difference network are important components of phased array antenna. In this paper, multilayer printed board is used to integrate millimeter wave multi-channel transceiver circuit and sum difference network. The interconnection between them is realized through RF coaxial vertical transition. At the same time, the heat dissipation design and inter channel shielding design of the module are carried out. The RF and low frequency required by the module are completed through the wiring between and within the dielectric plate layers. Finally, 128 arrays are fabricated and verified by multi-channel passive test. The results show that the type transceiver module integrating with two-dimensional sum difference network has good performance, and 128 channels have excellent amplitude and phase characteristics. The integration technology has the characteristics of lightweight, miniaturization, high integration and low manufacturing cost. It can be widely used in miniaturized phased array antennas.
\end{abstract}

\section{Keywords}

Multi-Channel Transceiver, Two-Dimensional Sum Difference Network, RF Coaxial Vertical Transition, High Integration

\section{Introduction}

With the development of wireless communication technology, the spectrum in the low frequency band has become more and more crowded. The demand for high-quality and high-capacity wireless communication equipment makes the communication frequency continuously improve. Millimeter wave can effectively solve many problems faced by high-speed broadband wireless access due 
to its wave length and wide frequency band. Therefore, it has a wide application prospect in short-range communication [1]. In order to improve the scanning speed and pointing accuracy, modern advanced radar and communication systems not only raise the working frequency band to the millimeter wave frequency band, but also abandon the traditional mechanical scanning platform and adopt phased array antenna to realize fast two-dimensional phased scanning [2]. The transceiver module (including transceiver circuit and multi-functional chip) and sum difference network are important components of phased array antenna. Its integration level determines the performance and cost of the whole system [3]. The transceiver module is used to amplify and shift the signal under the transceiver state of phased array antenna, and the power division and sum difference network is used to complete the power synthesis and distribution of the signal [4]. Generally, the transceiver module of phased array antenna is designed separately from the sum and difference network, which is an independent module [5]. The connection between modules is realized through the connection of connectors. This connection mode not only increases the cost of the circuit and system loss, but also has a large longitudinal size, which is not conducive to the miniaturization, lightweight and integrated design of the system [6]. With the development of phased array antenna in millimeter wave band, miniaturization and compactness are important requirements of phased array antenna. An integration technology is urgently needed to break the barriers between transceiver module and sum difference network, simplify the form of interconnection interface, reduce the manufacturing cost, and find a feasible method from processing and manufacturing [7].

\section{Basic Concepts}

Phased array antenna is divided into brick phased array and tile type phased array. Compared with brick phased array, tile type phased array has higher integration and smaller longitudinal size. It is suitable for platforms with limited installation space. Figure 1 shows the general structure of tile phased array antenna. In the figure, the antenna array, transceiver components and sum difference network are horizontally integrated and vertically connected. The vertical interconnection between layers is electrically connected through the upper and lower conduction of connectors.

In order to further improve the high-density and small volume integration requirements of the tile structure, the high-density integration of the transceiver

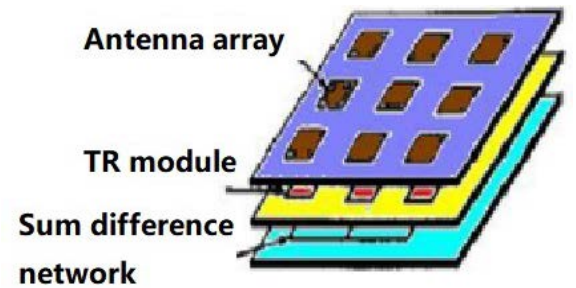

Figure 1. Overall structure of active tile type phased array. 
module and the sum difference network, as its core component, can not only greatly reduce the vertical height and reduce the space occupied by the interconnection between the transceiver module and the sum difference network, but also improve the circuit reliability by optimizing the low-frequency control and power supply routing of the transceiver module. As shown in Figure 2, the multi-channel transceiver circuit and sum difference network are designed on the same printed board. On the one hand, the routing of amplifier, phase shifter and power division network are on the same layer, and the RF signals are connected up and down through the RF vertical interconnection between PCB boards. On the other hand, the sum difference network, the control and power supply of transceiver circuit and other low-frequency signals are also arranged through PCB multilayer boards. Finally, the processed integrated transceiver circuit and the PCB multilayer board of sum difference network are welded on the metal substrate, and interconnected with the antenna array and wave control modules through buttons and other forms. At the same time, considering the working requirements of phase shifter and amplifier, an air chamber is reserved on the upper cover plate.

\section{Design Analysis}

The key points of the design are how to realize all the functions of the traditional $\mathrm{T} / \mathrm{R}$ module with small volume. As a kind of new structure, the three dimensional tile type $\mathrm{T} / \mathrm{R}$ module with fuzz button used in the microwave frequency can achieve good DC and microwave links, and does not need welding. In the traditional module, the microwave signal is transmitted into $\mathrm{X}-\mathrm{Y}$ in $2 \mathrm{D}$. In tile type $\mathrm{T} / \mathrm{R}$ module microwave signal transferred not only in $\mathrm{X}-\mathrm{Y}$ plane, but also in the $\mathrm{Z}$ axis direction. The use of $\mathrm{PCB}$ multi-layer wiring technology, through a reasonable layout, can realize good microwave performance. With metal isolation Cylinder, high frequency PCB multi-layer substrate can realize digital and analog signal isolation, and realize electromagnetic shielding to solve the problem of signal crosstalk. Three dimensional microwave signal simulation model of high frequency PCB substrate is shown in Figure 3 and Figure 4. The simulation results are obtained as shown in Figure 5 and Figure 6.

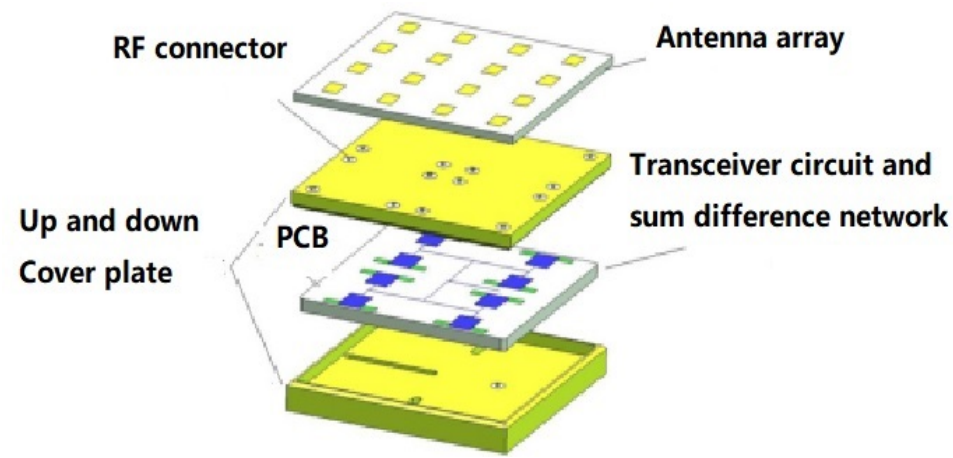

Figure 2. Schematic structure of tile type transceiver module integrating with two-dimensional sum difference network. 


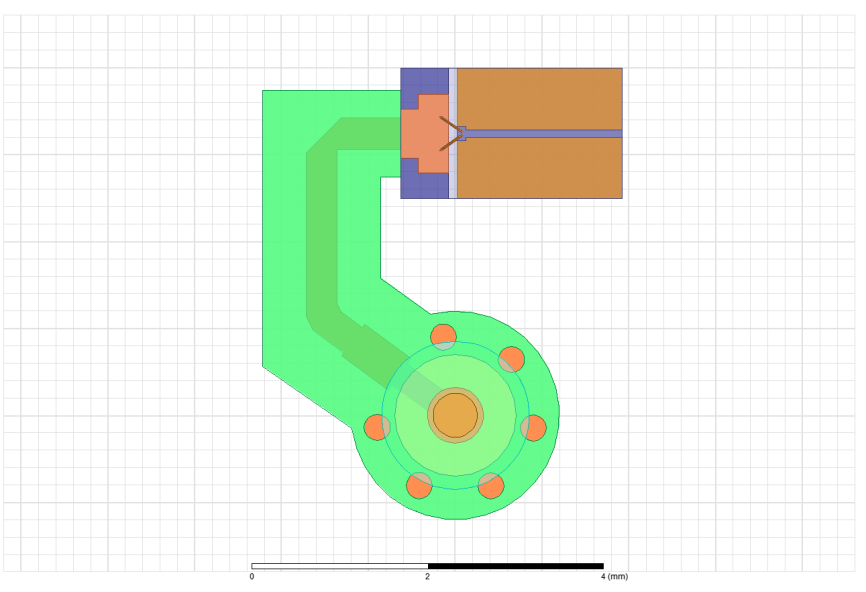

Figure 3. simulation model of the Micro-strip coaxial transition.

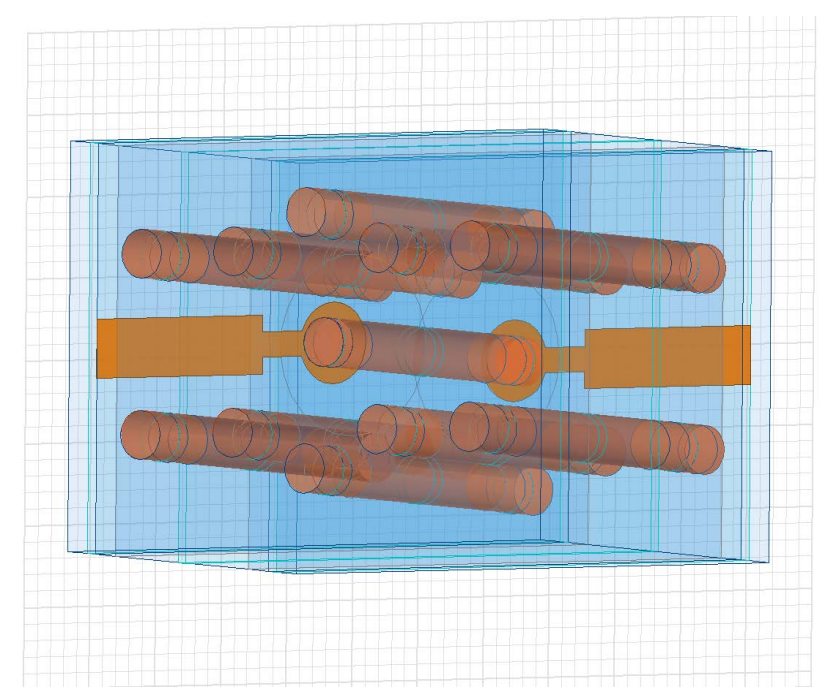

Figure 4. Simulation model of RF coaxial vertical transition.

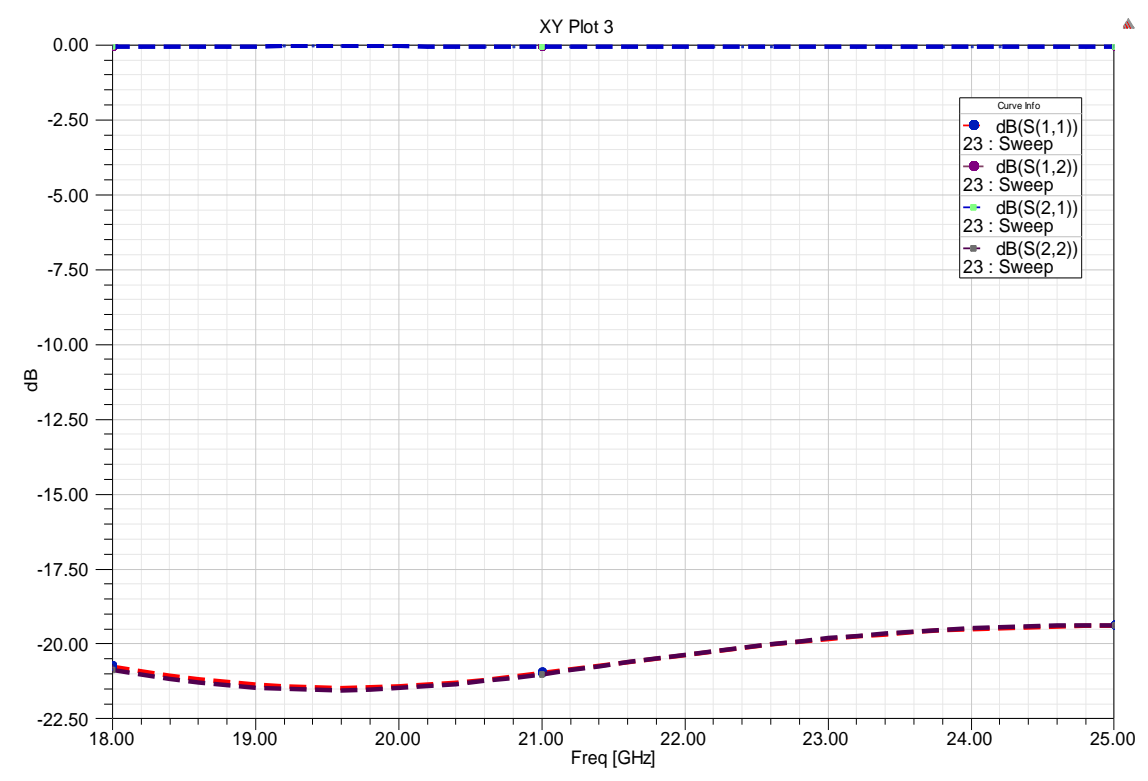

Figure 5. Simulation results of the above structure. 
From the simulation results we can see, in the $\mathrm{K}$ band, the transmission insertion loss of the vertical transition of the high frequency PCB substrate is less than $0.5 \mathrm{~dB}$, and the return loss is more than $20 \mathrm{~dB}$.

For the integration technology of multi-channel transceiver circuit and sum difference network, millimeter wave band $8 \times 16$ array is selected as an example for detailed design and analysis. Due to the small spacing of antenna units in millimeter wave band, the average area occupied by a single channel is about 7 $\mathrm{mm} \times 7 \mathrm{~mm}$, which makes the circuit routing of multi-channel transceiver circuit and sum difference network very compact. In this paper, the layout is in the form of unequal spacing. In order to reduce the cost, taconic TSM-DS3 is used as the printed board material in the design, as shown in Figure 7 and Figure 8. Among them, 1 is dielectric substrate, 2 is multi-channel transceiver chip, 3 is RF port connected with antenna, 4 and 9 is RF coaxial vertical transition, 5 is

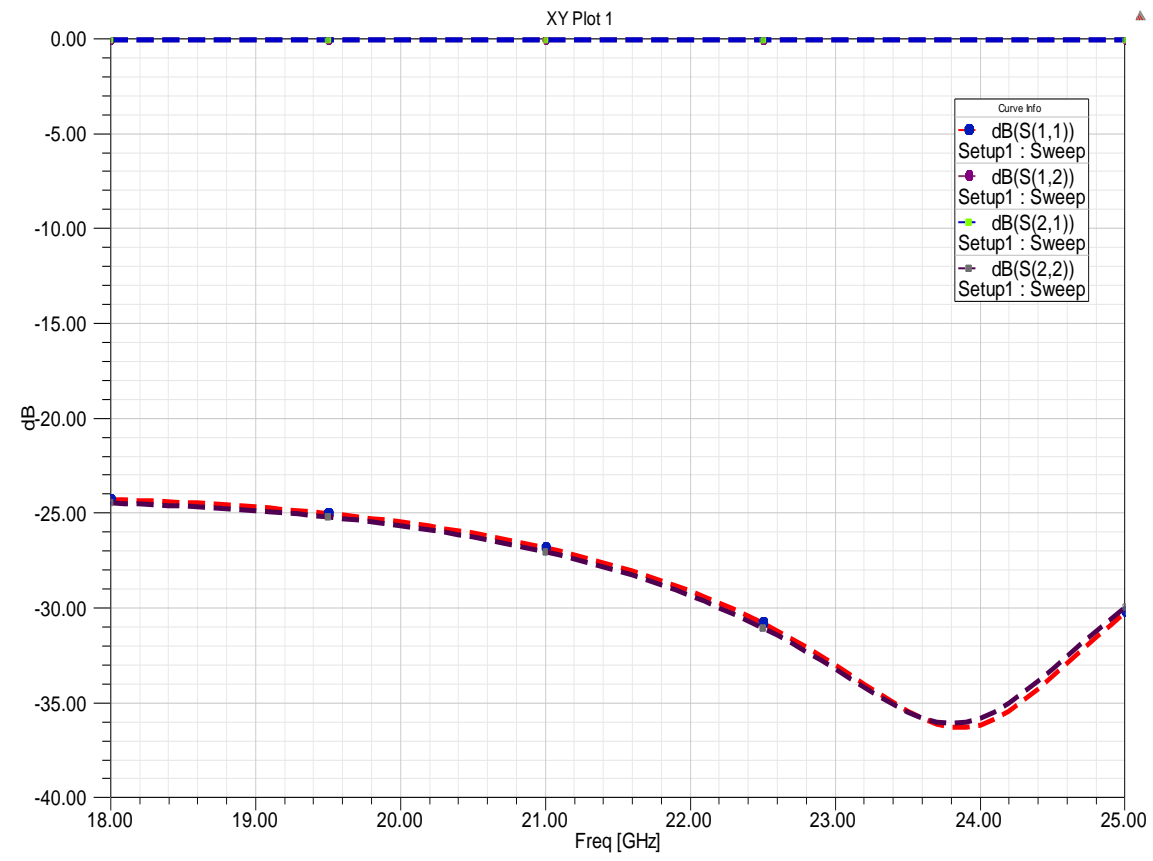

Figure 6. Simulation results of the vertical transition.

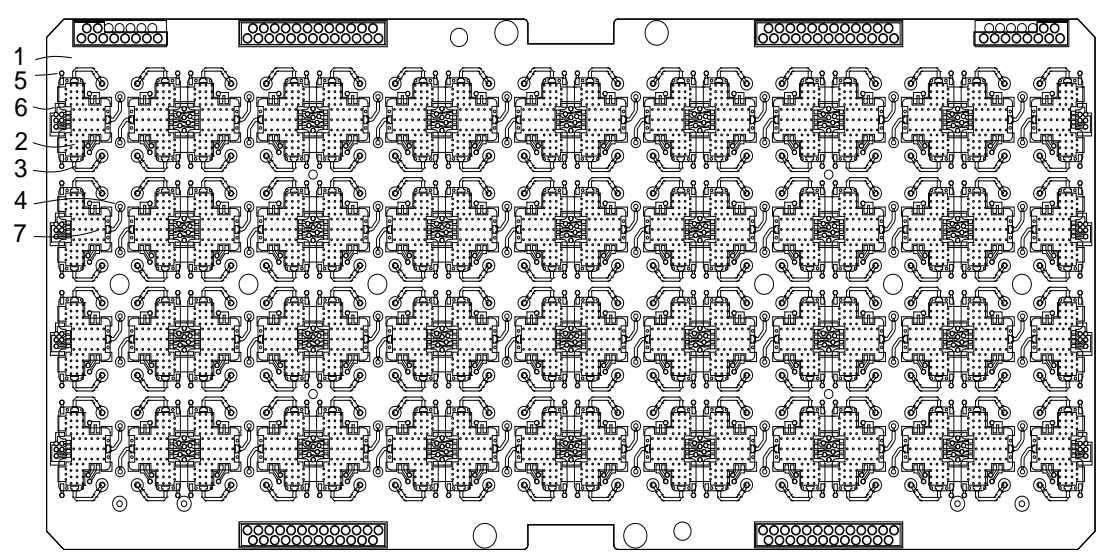

Figure 7. Upper surface of integrated multilayer printed board. 


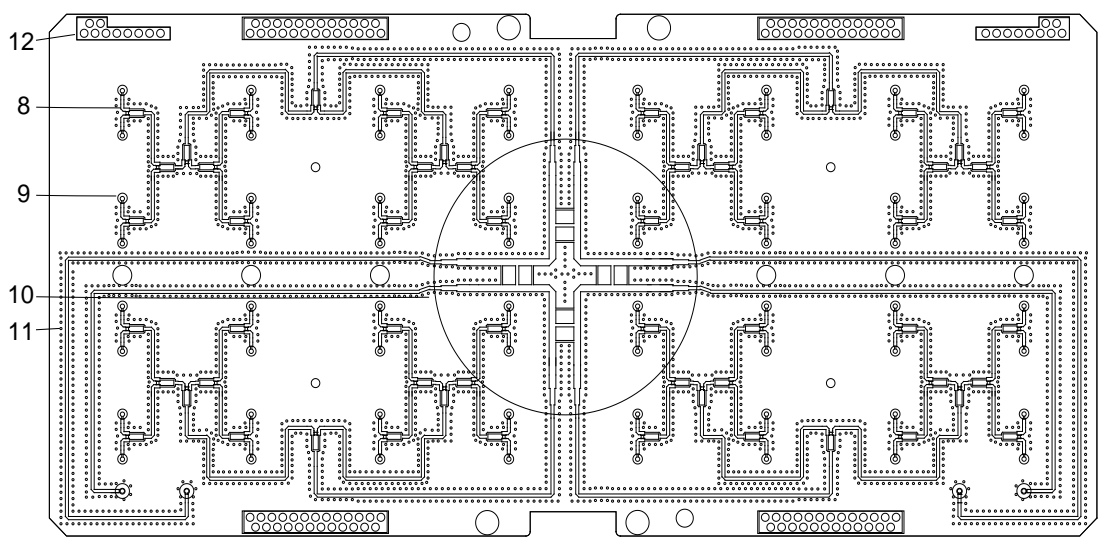

Figure 8. Sum difference Layer of the integrated multilayer printed board.

power supply pad, 6 is low-frequency control pad, 7 is Radiating hole, 8 is power division network, 10 is sum difference network, 11 is metal isolation column and 12 is low frequency pinhole.

As shown in Figure 8, the power distribution network and the sum difference network are located on the same layer and are isolated by metal isolation columns to reduce the impact between circuit wiring. The device mounting interface and the power distribution network are located in different layers, and they are vertically interconnected by the RF through hole on the board. The low-frequency control and power supply of the chip are connected with the pad near the chip through gold wire bonding. At the same time, a solid grounding column is designed under the chip installation interface, which not only provides grounding for the chip, but also acts as the heat dissipation channel of the chip to guide the heat to the bottom of the printed board.

\section{Test results and analysis}

Figure 9 shows the processed PCB multilayer substrate.

The millimeter wave band of $8 \times 16$ multi-channel transceiver circuit and sum difference network integrated circuit are processed, and the size is $114 \mathrm{~mm} \times 68$ $\mathrm{mm}$, the thickness is only about $3 \mathrm{~mm}$, and the weight of a single channel is about $2 \mathrm{~g}$. In order to verify the performance of the integrated circuit, the power divider is pasted at the corresponding position, and the RF fixture is designed for passive test. The test fixture and clamping diagram are shown in Figure 10. Because the RF interface of the board is a touch pad, in order to facilitate the test, one end of the designed RF fixture is a SMP, and the other end is an SSMP. During the test, the board is pressed in the middle of the fixture through screws, and the board is positioned with the fixture through pin holes. The instrument required for passive test is Agilent vector network analyzer n5244a. When testing one channel, the other 127 channels are terminated with matching load. The test results of 128 RF channels of the integrated circuit are shown in Figure 11 and Figure 12.

The statistical data table in Figure 6 is analyzed by "cascade test data analysis 

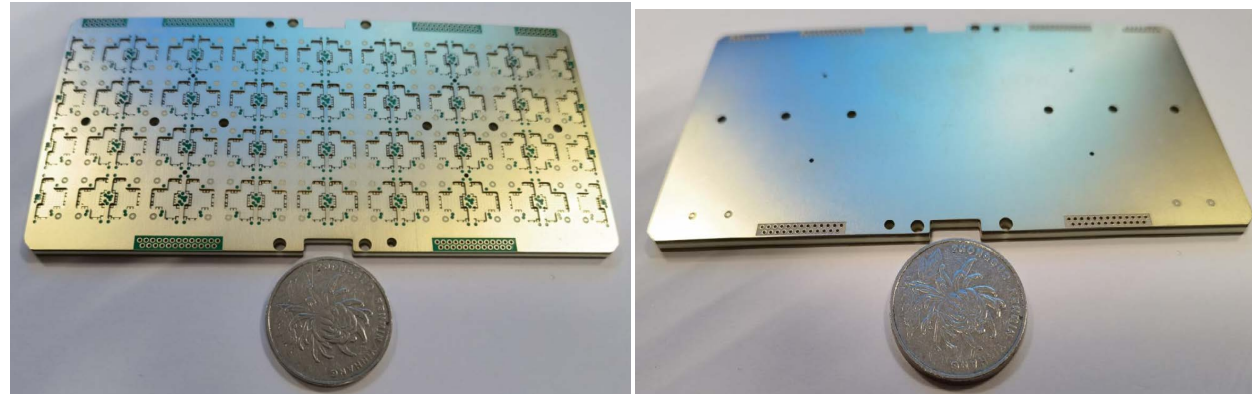

Figure 9. The high frequency PCB multilayer substrate.
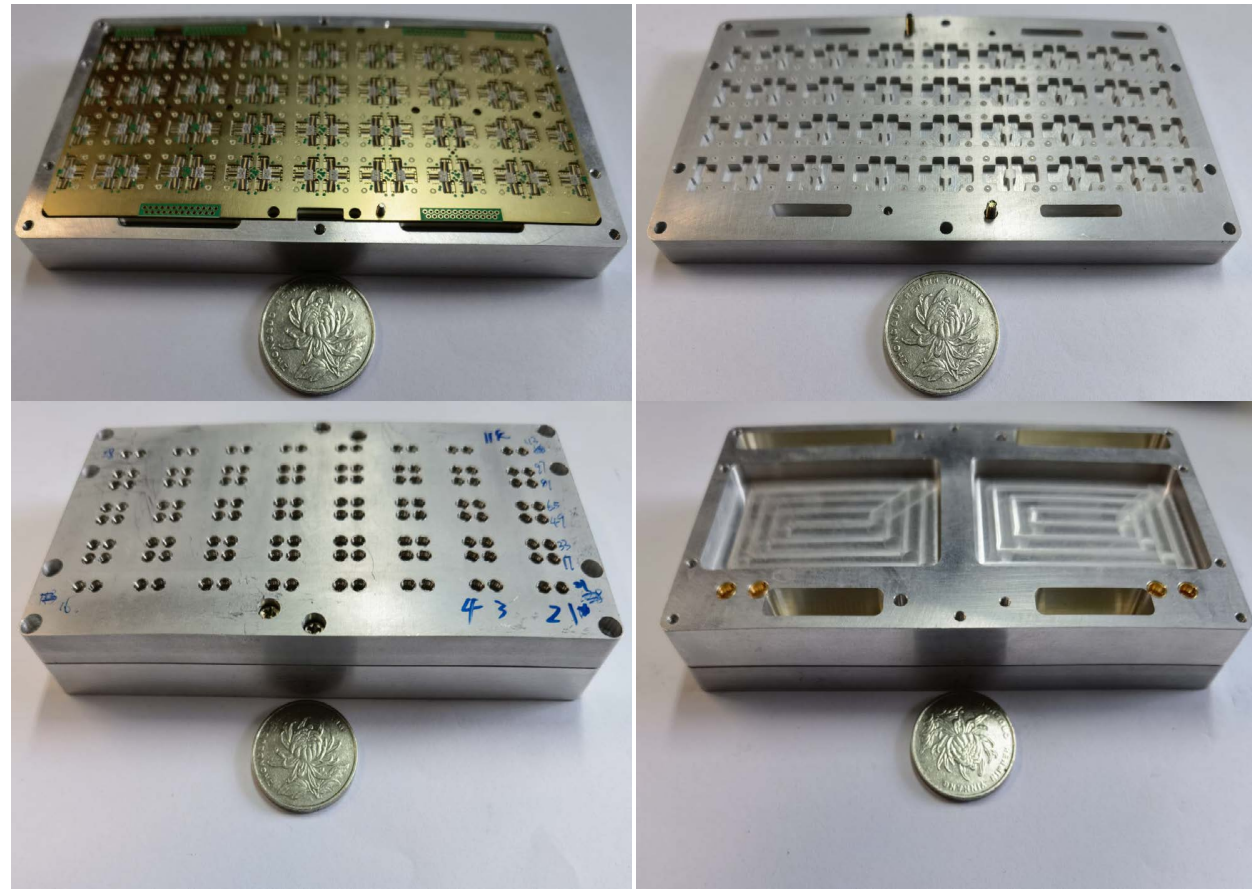

Figure 10. The tile type multi-channel transceiver module and the test fixture.
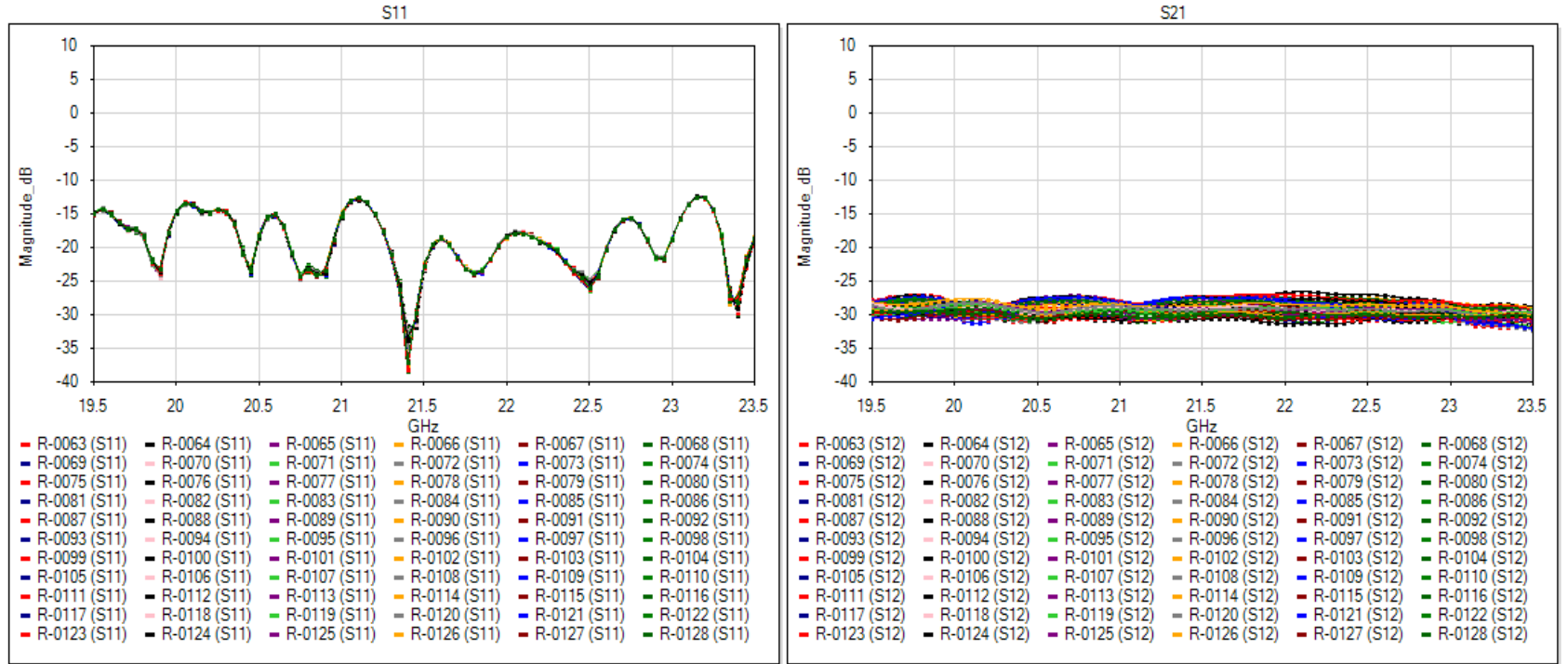

Figure 11. The Passive test results of each channel: $S 11(\mathrm{~dB})$ and $S 12(\mathrm{~dB})$. 

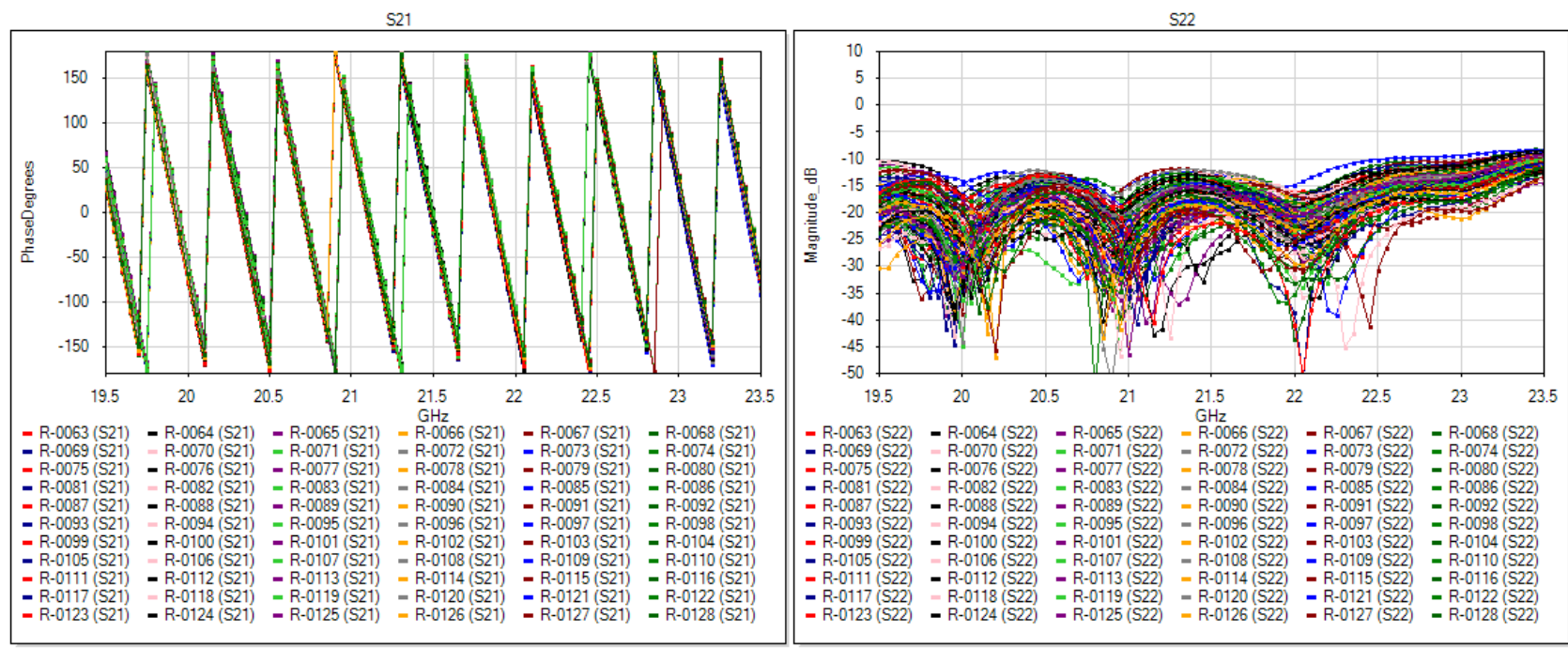

Figure 12. The Passive test results of each channel: S22 (dB) and S21 (phase).

software". Figure 11 shows the total port standing wave and the insertion loss of the channel. From the picture we can see $\mathrm{S} 11$ is better than $-12 \mathrm{~dB}, \mathrm{~S} 12$ is about $30 \mathrm{~dB}$ in $\mathrm{k}$ frequency band, and the amplitude consistency between channels is good. After deducting two test cables, the loss is about $27 \mathrm{~dB}$ (including distribution loss of $21 \mathrm{~dB}$ ). Figure 12 shows the split port standing wave and the phase of the channel. It can be seen that $\mathrm{S} 22$ is better than $-10 \mathrm{~dB}$, and the phase consistency between channels is good too. We come to the conclusion that the high-density integrated RF board works normally and has excellent amplitude and phase consistency between 128 channels. According to the statistics of MATLAB software, the RMS of amplitude and phase of 128 channels in the $\mathrm{k}$ frequency band are $0.5 \mathrm{db}$ and $5^{\circ}$. The test results show that the performance of the core part: multi-channel integrated circuit is normal, and the process scheme of microwave printed board is feasible. Compared with the performance indexes of single channel weight of about $30 \mathrm{~g}$, amplitude RMS $1.5 \mathrm{~dB}$ and phase RMS $10^{\circ}$ obtained by cascading the previous transceiver circuit and sum difference network independent module, the integrated module designed in this paper not only greatly reduces the weight, but also has significantly better performance, and improves the integration of tile type phased array.

\section{Conclusion}

The high-density integration technology of millimeter wave multi-channel transceiver circuit and sum difference network can not only achieve good channel electrical performance in millimeter wave band, but also improve the amplitude and phase consistency between channels. The high-density integration of multi-channel transceiver circuit and sum difference network greatly reduces the longitudinal height size and weight, provides an implementation way for the miniaturization and lightweight of phased array antenna, and also provides an important design idea for skin antenna technology in the future. At the same 
time, combined with the existing Microwave PCB processing technology, it is easy to process and realize, which is of great significance for engineering application.

\section{Conflicts of Interest}

The authors declare no conflicts of interest regarding the publication of this paper.

\section{References}

[1] Shao, C.S. (2016) Study Status and Development Trend of Phased Array Radar. Modern Radar, 38, 1-4. (In Chinese)

[2] Li, W.H., Cai, J.Y., Wang, Y.T., et al. (2015) The Operational Advantage, Performance Comparison and Military Application of Airborne APAR. National Defense Science \& Technology, 36, 69-73.

[3] Li, X. (2017) Design of an X Band Integrative T/R Module. Electronic Science and Technology, 30, 99-101.

[4] Yao, R.Y. and Wei, B. (2016) The Design of Ku-Band T/R Module. Electronics and Packaging, 16, 33-36.

[5] Vo, H.H., Chen, C.C., Hagan, P., et al. (2016) A Very Low-Profile UWB Phased array Antenna Design for Supporting Wide Angle Beam Steering. IEEE International Symposium on Phased Array Systems and Technology (PAST), Waltham, 18-21 October 2016, 483-490. https://doi.org/10.1109/ARRAY.2016.7832578

[6] Hu, C.M., Zhou, Z.P. and Yan, W. (2010) Phased Array Radar Transceiver Module Technology. National Defense Industry Press, Beijing.

[7] Ding, W.W., Zhao, W.P. and Mu, S.B. (2016) Research on T/R Module Technology of Active Phased Array Radar. Control and Guidance, 12, 77-83. 